\title{
Contribution à l'amélioration des pratiques de soins essentiels aux nouveau-nés dans le centre de communautaire et universitaire (CSCom U) de Konobougou
}

\section{Contribution to the improvement of essential care practices for newborns in the community and university center (CSCom U) of Konobougou}

\author{
Coulibaly $\mathrm{D}^{1^{*}}$, Coulibaly $\mathrm{MB}^{2}$, Goïta $\mathrm{S}^{3}$, Niaré $\mathrm{B}^{4}$, Diakité $A A^{5}$, Dicko $F^{3,5}$
}

\section{DOI : 10.53318/msp.v11i1.1900}

$1:$ Centre de santé de référence de Bla (Ségou-Mali)

2 : Centre Santé Communautaire et Universitaire de Konobougou (Ségou-Mali)

3 : Département de médecine de famille/ médecine communautaire à la FMOS (Bamako-Mali)

4 : Direction Régionale de la Santé de Bamako (Bamako-Mali)

5 : Service de pédiatrie au CHU de Gabriel Touré (Bamako- Mali)

*Correspondant : Dr Djénèba Coulibaly ; Médecin de famille/médecin communautaire au Centre Santé de Référence de Bla ; tel : 0022374 $\begin{array}{llllllllll}51 & 74 & 55 / & 00 & 223 & 69 & 10 & 29 & 11 & \text {; mail : }\end{array}$ tomdolar@yahoo.fr/tomdolar95@gmail.com

\section{Résumé}

Introduction : La période postnatale immédiate est une étape fondamentale d'adaptation physiologique et psychologique pour le nouveau-né. La plupart des difficultés d'adaptation à la vie extra-utérine sont prévisibles et doivent être anticipées. Le but de cette étude était de contribuer à l'amélioration des pratiques de soins essentiels aux nouveau-nés dans le centre de santé communautaire et universitaire (CSCom-U) de Konobougou. Matériel et méthodes : II s'agissait d'une étude de recherche action qui s'est déroulée dans la maternité du CSCom U de Konobougou dans le district sanitaire de Barouéli/Région de Ségou du 01 décembre 2020 au 31 janvier 2021. Nous avons introduit dans cette étude 32 mères et 8 agents de santé. Résultats : Les matrones étaient plus représentées avec $62,5 \%$ suivies des sages-femmes avec $25 \%$. Avant l'enquête tous les matériels étaient disponibles sauf la sonde nasogastrique. Suite à notre recommandation l'équipe a décidé de s'en procurer une, ce qui a permis de porter la disponibilité des matériels à 100\%. A la phase initiale de l'étude, $25 \%$ des mères enquêtées avaient reçu des consignes sur les soins du cordon ombilical, suite à la mise à niveau des prestataires nous avons obtenu une augmentation de $75 \%$ à la phase finale soit $100 \%$. Conclusion : Les soins essentiels du nouveau-né restaient insuffisants pour déficit de formation initiale et/ou continue d'une part et pour instabilité du personnel d'autre part. Ainsi, nous avons recommandé une mise en place de programme de formation continue et de suiviévaluation destiné aux prestataires.

Mots clés : Soins essentiels, nouveau-nés, centre de communautaire et universitaire, Konobougou

\section{Abstract}

Introduction: The immediate postnatal period is a fundamental stage of physiological and psychological adaptation for the newborn. Most difficulties in adapting to extra-uterine life are predictable and should be anticipated. The aim of this study was to contribute to the improvement of essential care practices for newborns in the health community and university center (CSCom-U) of Konobougou. Material and methods: This was an action research study which took place in the maternity unit of the CSCom U of Konobougou in the health district of Barouéli / Region of Ségou from December 01, 2020 to January 31, 2021. We included in this study 32 mothers and 8 health workers. Results: Midwives were more represented with $62.5 \%$ followed by midwives with $25 \%$. Before the survey all materials were available except the nasogastric tube. Following our recommendation, the team decided to procure one, which increased the availability of materials to $100 \%$. At the initial phase of the study, $25 \%$ of the mothers surveyed had received instructions on umbilical cord care, following the upgrading of providers we obtained an increase of $75 \%$ in the final phase. Conclusion: Essential newborn care remained insufficient, due to a lack of initial and / or continuing training on the one hand and staff instability on the other. We therefore recommended setting up a continuous training and monitoring-evaluation program for service providers.

Keywords: Essential newborn care, community and university center, Konobougou, Mali

\section{Introduction}

La période postnatale immédiate est une étape fondamentale d'adaptation physiologique et psychologique pour le nouveau-né (1). Le nouveau-né à terme est indemne de pathologie, dispose des ressources anatomiques et physiologiques adéquates pour s'adapter à la vie aérienne (2). La plupart des difficultés d'adaptation à la vie extra-utérine sont prévisibles et doivent être anticipées (3).

La réduction de la mortalité infanto-juvénile passe par celle de la mortalité néonatale qui reste très élevée dans certains pays. La réduction de la mortalité néonatale passe par l'amélioration des compétences des prestataires en salle d'accouchement (4).

Au plan mondial, le nombre de décès néonatal est passé de 5,1 millions en 1990 à 2,6 millions en 2016 (5). La mortalité néonatale précoce qui découle des difficultés à la naissance varie de $2,8 \%$ dans les pays développés à $26,5 \%$ dans les pays en voie de développement particulièrement en Afrique (3). En effet, en Afrique subsaharienne, elle reste encore très élevée. Ainsi, on note un taux de $42 \%$ en République centrafricaine, 38\% 
en Somalie, 38\% en Lesotho, 38\% en Guinée -Bissau, $37 \%$ au Soudan du Sud, $36 \%$ en Côte d'Ivoire, 35\%o au Tchad (5).

Au Mali, malgré les efforts du gouvernement et de ses partenaires pour former les professionnels de santé en soins essentiels aux nouveau-nés (SENN) le taux de mortalité néonatale reste élevé (6). Selon, l'Enquête démographique de Santé Mali VI (EDSM-VI) en 2018, le taux de mortalité infantile est de 54 pour 1000 naissances vivantes ; le taux de mortalité néonatale est de 33 pour 1 000 naissances vivantes (7).

Les soins recommandés pour tout nouveau-né s'alignent sur les standards internationaux basés sur les données factuelles et les recommandations de l'Organisation mondiale de la santé (OMS). Ils sont appelés SENN et comprennent (8) : Soins immédiats à la naissance ; Soins au nouveau-né au cours des six premières heures ; Soins au nouveau-né de 6 heures jusqu'à 6 semaines.

Dans une étude réalisée au Mali sur l'évaluation du niveau de connaissance des professionnels de santé à Bamako (2016), il est ressorti que le niveau de connaissance des professionnels de santé sur les SENN est faible (6). Cette faiblesse serait à l'origine de la non-application des SENN dans les centres de santé concernés, ce qui pourrait contribuer à la non-réduction du taux de mortalité néonatale. L'étude recommande, par conséquent, l'intégration des SENN dans les programmes de formation initiale des professionnels de santé ainsi que leur formation continue en la matière (6).

$\mathrm{Au}$ Centre de Santé Communautaire et Universitaire (CSCom-U) de Konobougou, selon les données du logiciel de gestion de l'information sanitaire de District version deux (DHIS2), nous avons enregistré, en 2019, 3 cas de mort-nés frais et 11 cas de mort-nés macérés, soit 2,3\% de mortalité néonatale précoce ; 8 cas de réanimation du nouveau-né et 28 cas de petit poids de naissance pour un total de 607 naissances vivantes. Konobougou ne fait donc pas exception des problèmes liés à la survie des nouveau-nés.

Ainsi, nous avons mené ce travail dans la maternité du centre de santé communautaire et universitaire de Konobougou afin de contribuer à l'amélioration des pratiques de soins essentiels aux nouveau-nés.

\section{Matériel et méthodes}

II s'agissait d'une étude de recherche action (transversale répétée et interventionnelle). Elle s'est déroulée dans la maternité du CSCom-U de Konobougou dans le district sanitaire de Barouéli dans la Région de Ségou durant deux mois allant du 1er au 31 décembre 2020 au 31 janvier 2021.

Elle concernait le personnel de la maternité impliqué dans l'accouchement et les mères d'enfants ayant donné leur accord pour participer à l'étude et qui ont accouché au CSCom-U. N'étaient pas inclus dans cette étude les agents de santé travaillant dans les autres unités de soins dans le CSCom-U de Konobougou et les femmes accouchées à domicile mises en observation au centre pour complication obstétricale. Le nombre total de personnes incluses était de 32 pour les mères et 8 pour le personnel. Les 16 mères enquêtées à la première évaluation n'étaient pas la même la seconde évaluation. Mais pour le personnel, c'était les mêmes personnes. Nous avons procédé à un échantillonnage aléatoire systématique : les unités statistiques sélectionnées ont été espacées régulièrement au sein de cette population : chaque deuxième. L'unité statistique de la population était sélectionnée jusqu'à constituer la taille de l'échantillon fixé à 16 pour chaque évaluation soit 32 femmes pour les deux évaluations.

L'étude était composée de quatre étapes. La 1 ère étape pour la prise de contact avec les autorités socio-sanitaires associées au personnel impliqué à savoir : le président ASACO, le directeur technique du centre (DTC), les sages-femmes, l'infirmière obstétricienne et les matrones au cours de laquelle, l'explication du contexte de l'étude a été faite. Une première évaluation a été faite pour déterminer l'état de lieu. La 2ème étape pour l'élaboration d'un plan d'action dans la résolution des problèmes identifiés par la première évaluation. Cette phase consistait à ressortir les points forts et les points à améliorer tout en identifiant les goulets d'étranglement, avec le personnel impliqué. II s'agissait concrètement de s'interroger (chercheur, DTC et personnel de la maternité, membres de l'ASACO) sur l'origine de l'insuffisance observée et de faire un plan d'action. La 3ème étape était consacrée à la mise en œuvre du plan d'action : dans cette phase Nous avons mis en œuvre l'ensemble des stratégies correctrices. La méthodologie adoptée était tout d'abord une formation des acteurs impliqués au cours d'une journée de rencontre. La 4ème étape concernait l'évaluation des actions menées pendant la troisième phase.

Nous avons collecté les données de la même manière au cours des deux évaluations en observant le personnel pendant la pratique des soins essentiels au nouveau-né. Une grille d'évaluation pour les infrastructures et le matériel des soins essentiels du nouveau-né a été introduite. Un questionnaire a été soumis aux mères. Les données ont été saisies et analysées (une comparaison descriptives a été faite entre les résultats de la première et ceux de la deuxième évaluation) sur épi info version 7. L'étude s'est déroulée en respectant les règles d'éthique liées à la recherche sur les personnes. II s'agissait d'une étude permettant d'apporter des solutions aux problèmes observés dans la pratique de soins essentiels aux nouveau-nés. Un consentement individuel écrit et signé a été obtenu de chaque participant à l'étude.

\section{Résultats}

La première évaluation a permis de dégager des points forts et à améliorer. A la suite de cela, il a été proposé de : 
- Organiser des journées de rencontre sur les SENN pour corriger les insuffisances observées à travers des propositions de solutions ;

- Observer les prestataires au cours de la prise en charge et du suivi de nouveau-nés pour les aider à un changement de comportement et un renforcement de compétences ;

- Renforcer la sensibilisation sur les SENN.

Ces points cités en haut ont été réalisées avant

l'évaluation finale.

Le coin du nouveau-né était disponible pendant les deux évaluations. Le manuel de la politique des SENN était disponible tout au long de l'étude. A la première évaluation, tout le matériel était disponible sauf la sonde nasogastrique (SNG). Tous les intrants et le personnel étaient disponibles à l'évaluation initiale et finale. Les matrones étaient plus représentées avec cinq personnes suivies des sages-femmes au nombre de deux.

A la première évaluation, six agents de santé sur huit avaient déjà reçu une formation sur les SENN. Entre les deux évaluations, tout le personnel a été formé. (Tableau I). Trois agents de santé avaient une expérience professionnelle de 1 à 5 ans suivis de deux agents de santé avec 20 ans et plus d'expérience (Tableau II).

A la première évaluation on a constaté une bonne maîtrise des étapes 1 à $5,7,8$ et 10 . Toutefois, nous avons noté une insuffisance chez un certain nombre d'agents pour les étapes (étape 6 : contact peau à peau avec la mère était maitrisé par deux agents de santé, étape 9 : Administration de vitamine $\mathrm{K} 1$ était aussi maitrisé par deux agents de santé, et 11 : Initiation précoce de l'allaitement maternel était réalisée par quatre agents de santé). La formation du personnel sur les SENN a permis une bonne maîtrise de toutes les étapes (Tableau III).

A la première évaluation, nous avons remarqué une insuffisance importante dans les soins au nouveau-né au cours des six premières heures. Aucun prestataire de l'effectif ne vérifiait les cinq premiers paramètres, et seulement deux d'entre eux procédaient à l'examen complet du nouveau-né avant la sortie. Après notre intervention, tout l'effectif a eu une bonne maitrise de vérifier tous les paramètres durant les six premières heures (Tableau IV).

Plus de la moitié des mères enquêtées soit dix mères étaient dans la tranche d'âge de 20 - 24 ans pendant les deux évaluations de l'étude, suivie de six mères entre 25 et 49 ans à l'évaluation initiale et quatre mères à la seconde évaluation (Tableau $\mathrm{V}$ ). Dans la première évaluation, neuf mères avaient un niveau d'étude secondaire, suivies de non scolarisée soit sept mères. Dans la deuxième évaluation, les non scolarisées étaient fortement représentées soit huit mères, suivies de niveau d'étude primaire soit quatre mères. A la première évaluation, douze mères étaient ménagères contre dix mères à l'évaluation finale. Les trente deux femmes étaient mariées.

A la première évaluation, seuls dix des mères enquêtées avaient reçu des consignes sur la prévention des infections contre seize après l'intervention. A la première évaluation de l'étude, quatre mères enquêtées avaient reçu des consignes sur les soins du cordon ombilical contre seize mères suite au plan d'action. Toutes les mères enquêtées avaient reçu des consignes sur le bain du nouveau-né par le personnel au cours des deux évaluations. À l'évaluation initiale, dix mères avaient reçu des consignes sur l'allaitement maternel exclusif (AME) contre seize à la seconde évaluation. Au cours de l'évaluation primaire, aucune mère enquêtée n'avait reçu des consignes sur les signes de danger. Après l'intervention toutes les mères ont reçu des consignes (Tableau VI). À l'évaluation initiale, quatre mères enquêtées avaient reçu des consignes sur le calendrier vaccinal contre seize à la seconde évaluation.

\section{Discussion}

Le progrès du résultat dans la deuxième évaluation était lié à des journées de rencontre et de la formation avec le personnel soignant et les accompagnements que nous avons effectué, le tout associé à l'accentuation de la sensibilisation. Le coin du nouveau-né était disponible pendant toute la période de l'étude, mais n'était pas utilisé par les accoucheuses. L'utilisation de ce coin permet de garder le nouveau-né à chaud pour lui permettre de ne pas tomber en hypothermie.

A la première évaluation, tous les matériels étaient disponibles sauf la sonde nasogastrique (SNG). Suite à la recommandation de l'enquêteur l'équipe a décidé de s'en procurer une, ce qui a permis de rendre disponible la totalité des matériels à qui étaient les suivants : Pèse bébé, toise, thermomètre, horloge, Stéthoscope, ballon avec masque, Sonde nasogastrique, matériel d'aspiration, ciseaux propre et stériles et la poire. Le manuel de la politique des SENN était disponible tout au long de l'étude. Dans la totalité des cas les intrants étaient disponibles pendant les deux évaluations à savoir : la vitamine $\mathrm{K} 1$, la tétracycline $1 \%$, la Chlorhexidine, le VPO et le BCG. La disponibilité de ces matériels permettaient de faire une bonne prise en charge des nouveau-nés en particulier ceux ayant des difficultés d'adaptation.

Au cours des deux évaluations, les matrones étaient majoritaires suivies des sages-femmes qui représentaient le quart. Cela était dû à l'insuffisance du personnel qualifié en milieu rural. Tandis que les sages-femmes étaient les plus représentées avec $34,1 \%$ suivies des Infirmiers avec $31,7 \%$ selon l'étude de Mariko D en 2018 à Bamako (13). À l'évaluation initiale, les trois quarts du personnel avaient déjà reçu une formation sur les SENN. Cette situation était mieux appréciée par rapport à d'autres ou le taux de formation ne dépasse pas les $60 \%$. C'est ce qui ressortait de l'étude de Boiro $D$ et al. qui notait que plus de $59 \%$ des prestataires n'étaient pas formés en soins essentiels au nouveau-né en 2016 à Dakar (10). Tandis que plus de la moitié du personnel soit $55,3 \%$ n'avait pas reçu de formation sur les SENN selon l'étude de Mariko D (13). Suite à la formation que nous avons organisée pendant l'étude tout le personnel a été formé. Ainsi notre 
intervention a permis une augmentation de $25 \%$ en termes de formation.

Dans notre étude moins de la moitié des enquêtées avait une expérience professionnelle de 1 à 5 ans et un quart se plaçait dans la catégorie de plus de 20 ans expérience. Le reste des tranches d'âge (6-10 ans, 11-15 ans et 16-20 ans) en termes d'expérience professionnelle était légèrement supérieur au dixième pour chacun. Alors que dans l'étude de Boiro $D$ et al. plus de la moitié des prestataires enquêtés avait une expérience supérieure à 6 ans dans la santé ; $34 \%$ entre 2 et 5 ans et $7 \%$, une année ou moins (10). Par contre selon Mariko D, 41\% des enquêtées avaient une expérience professionnelle comprise entre 1 et 5 ans (13).

Au cours des deux évaluations, la préparation pour la naissance était globalement maitrisée, car les prestataires s'assuraient que toutes les surfaces avec lesquelles le bébé entre en contact soient propres, sèches et bien éclairées et chaud sans courant d'air, elles vérifiaient la disponibilité, la propreté/stérilité et la fonctionnalité du matériel y compris pour le matériel de réanimation. Elles portaient une blouse et autres équipements de protection, elles utilisaient le savon liquide ou solide, la source d'eau propre et les gants stériles. Cela permettait d'être rapide et d'éviter la perte du temps en cas de besoin urgent pour la réanimation d'une part et permettait la prévention des infections d'autre part. Le niveau d'hygiène paraissait satisfaisant. S'agissant de la prévention des infections, l'hygiène des mains est un indicateur de qualité mettant en évidence la sécurité des systèmes de soins. «Un soin propre est un soin plus sûr ». Dans le cadre des soins de santé, l'hygiène des mains a sauvé des millions de vies ces dernières années selon Njom Nlend AE (14). Mais le niveau d'hygiène ne se ressent pas en pratique car les infections sont au premier plan des causes de mortalité néonatale. Paradoxalement, Dicko-Traore et al. (15) montraient qu'il existait un manque réel d'information du personnel sur l'hygiène des mains dans le service de néonatologie et soins intensifs de l'hôpital Nord-Marseille mais les infections ne dominaient pas pour autant les causes de la mortalité.

Au cours des deux évaluations, le séchage était bien maitrisé, le personnel essuyait les sécrétions sur le visage, la bouche et le nez, si nécessaire. II séchait le bébé immédiatement et entièrement, et se débarrassaient du linge mouillé. Pendant le séchage, il vérifiait la respiration et la couleur du bébé. Ces gestes permettaient de libérer les voies aériennes du nouveau-né d'éviter une hypothermie.

Les soins du cordon ombilical étaient bien connus par la totalité des prestataires, elles attendaient jusqu'à ce que le cordon cesse de battre avant de le clamper et de le couper. Elles clampaient à $5 \mathrm{~cm}$ de l'abdomen du bébé, elles plaçaient une deuxième pince à $2 \mathrm{~cm}$ du côté maternelle et sectionnaient le cordon entre les 2 pinces avec une paire de ciseaux stériles, elles mettaient le clamp de Bar et appliquaient une solution antiseptique (Chlorhexidine) sur le bout et à la base du cordon. Le respect de la technique du clampage permettait d'éviter l'hémorragie du nouveau-né et de l'anémie. Dans une étude, Njom Nlend et al. trouvent que le clampage du cordon était pratiqué à l'immédiat dès la naissance dans la plupart des structures enquêtées à $95 \%$; les soins du cordon utilisaient des antiseptiques essentiellement la Chlorhexidine et l'alcool (14). Par contre celle de Jyoti Sarin et al. en Inde qui montrait que les soins du cordon étaient maitrisés à $61,3 \%$ (16).

Les soins des yeux étaient également bien connus par la totalité des prestataires. Elles tenaient un œil ouvert en séparant doucement les paupières supérieure et inférieure, mettaient un filon de pommade tétracycline $1 \%$ de l'angle interne vers l'angle externe en logeant l'intérieur de la paupière inférieure. Elles utilisaient la même technique pour l'autre œil. Nos résultats étaient satisfaisants comparés à ceux de Jyoti Sarin et al. en Inde qui montraient que les soins oculaires étaient maitrisés à $38,70 \%$ (16).

Au cours des deux évaluations, la totalité des prestataires avait identifié le bébé. Cette pratique est bonne car permettait de ne pas confondre les bébés.

Au cours de la première évaluation, seul un quart des prestataires maitrisait le maintien au chaud en peau à peau avec sa mère. Ailleurs selon l'étude de Boiro $D$ et al. qui montrait que $6 \%$ des prestataires avaient une bonne maitrise de maintenir le nouveau-né peau à peau avec sa mère (10).

Au cours de la première évaluation, seul un quart de personnel administrait $1 \mathrm{mg}$ de vitamine $\mathrm{K} 1$ en $\mathrm{IM}, 75 \%$ administraient par voie orale. Ces résultats étaient similaires à ceux de Boiro $D$ et al. qui montraient $32 \%$ de bonne maitrise sur l'utilisation de la vitamine K1 (10).

Par rapport à la mensuration, la totalité des prestataires avait une bonne maitrise à l'évaluation initiale et secondaire.

A la première évaluation, environ la moitié des nouveaunés avait bénéficié la mise au sein précoce. Nos résultats étaient similaires à ceux de Mariko $\mathrm{O}$ au Mali, qui avaient trouvé $59,5 \%$ des nouveau-nés qui avaient bénéficié d'une mise au sein juste les 30 min après l'accouchement (17). Dans l'étude de Gamgne BK au Cameroun, seulement 7,6 $\%$ des nouveau-nés avaient bénéficié la mise au sein précoce (18). Ceux de Boiro D et al. qui montraient $20 \%$ des nouveau-nés avaient bénéficié la mise au sein précoce (10).

Suite à la formation que nous avons organisée, une amélioration globale a été observée à toutes les étapes. Nos résultats étaient similaires de celui de Monebenimp $F$ au Cameroun où les soins immédiats étaient aussi bien maitrisés à $100 \%$ (19).

A la première évaluation, nous avons remarqué une insuffisance dans les soins au nouveau-né au cours des six premières heures. Seulement un quart des prestataires a fait l'examen complet du nouveau-né. Après notre intervention nous avons obtenu une bonne amélioration dans la totalité des cas. Les nouveau-nés étaient oubliés dans la maternité. Aucun prestataires n'avaient suivi les 
paramètres à savoir : La température, la respiration ; la coloration, le saignement du cordon, la technique l'allaitement maternel.

Plus de la moitié des mères enquêtées se situait dans la tranche d'âge de 20 et 24 ans dans les deux évaluations de l'étude, suivie de 25 et 49 ans avec respectivement plus du quart à l'évaluation initiale et le quart à la seconde évaluation.

Dans la première évaluation, un peu plus de la moitié des mères enquêtées avait un niveau d'étude secondaire. Dans la deuxième évaluation, les non scolarisées représentaient la moitié, suivie de niveau d'étude primaire qui représentait le quart. Ailleurs au Benin le niveau d'étude des mères étaient non scolarisé dans $56,8 \%$ des cas (19).

Dans la première évaluation, les mères enquêtées avaient une bonne maitrise sur le bain du nouveau-né, une insuffisance sur la prévention des infections avait été notée dans plus de la moitie des cas, les soins du cordon ombilical était connu dans un quart des cas, l'AME était connu dans moins des trois quarts des cas, les signes de danger étaient méconnus dans la totalité des cas et la vaccination connue dans un quart des cas. Après l'intervention toutes les mères avaient reçu des consignes avant la sortie à la maternité. Cela signifiait que les consignes étaient insuffisamment donnés à ces mères avant cette étude, le plan d'action a permis de donner des informations à toutes femmes et surtout des renseignements clairs.

\section{Conclusion}

II ressortait de l'analyse que les soins essentiels du nouveau-né restent insuffisants. Ceci semblait être expliqué par un déficit de formation initiale et/ou continue d'une part et l'instabilité du personnel d'autre part. Ainsi, nous recommandons une mise en place de programme de formation continue et de suivi-évaluation destiné aux prestataires s'occupant des soins du nouveau-né dans la maternité du centre de santé communautaire et universitaire de Konobougou.

\section{Conflit d'intérêt : aucun}

\section{Références}

1. Pejoan $\mathbf{H}$. Les recommandations et les consensus en cours concernant les soins au nouveau-né bien portant en salle de naissance. La revue Sagefemme. $2010 ; 9$ : 189-194.

2. Collège National des Sages-femmes. La Sagefemme et le nouveau-né : compétences et bonnes pratiques. La quatrième édition de la « Revue sagefemme. Paris, 2012, 25p.

3. Kinda $B$, Ouédraogo $S$, Koné $A$, Koueta $F$, Simporé A, Kaboré F. Détresse vitale du nouveauné en salle de naissance au CHU-YO de Ouagadougou : intérêt de la réanimation néo natale. The Pan African Medical Journal. 2016 ; 23 : 234
4. Azoumah K D, Agbeko F, Douti K N, Segbedji K A R, Tchagbele O B, Geraldo A. Évaluation des compétences des prestataires de soins exerçant dans les maternités en matière de réanimation néonatale au Togo. Revue de medecine perinatale. $2015 ; 7: 245$

5. Groupe inter organisations des Nations Unies pour l'estimation de la mortalité juvénile, 2017, statistiques mondiales de l'OMS sur les personnels de santé, 2016.

http : //www.who.int/hrh/statistics/hwfstats/en/, consulté le 21 février 2020.

6. Traoré $\mathbf{F} \mathbf{D}$, Sylla $\mathbf{M}$, Diall $H$, Traoré $\mathbf{M}$, Togo $\mathbf{P}$, Maïga $\mathbf{M}$ et al. Knowledge of Health Professionals on Essential Newborn Care in Bamako, Mali. Open Journal of Pediatrics $2018 ; 8: 311-323$

7. Ministère de la santé du Mali. Enquête Démographique et de Santé Mali Gème phase (EDSM VI) ; 2018. p 147-148.

8. Politique Normes et Procédures. Procédures en santé de la reproduction. Survie de l'enfant de 0 à 5 ans. Volume 4, juin 2019, $6 \mathrm{p}$.

9. Gabriel G, Lessard-Hébert $M$. La recherche action : Ses fonctions, son fondement et son instrumentalisation. Presse de l'Université de Québec. $1987 ; 125 p$.

10. Boiro D, Gueye M, Fattah M, Kane A, Ndongo A A, Thiongane A. Evaluation des connaissances sur les soins essentiels du nouveau-né (SENN) et la prévention des infections chez le personnel de sante en maternité à Dakar. Rev.CAMES SANTE. 2016 ; $4: 63-67$

11. Bee M, Shiroor A, Hill Z. Neonatal care practices in sub-Saharan Africa: a systematic review of quantitative and qualitative data. J Health Popul Nutr. $2018 ; 37(1): 9$.

12. Adejuyigbe E A, Bee M H, Amare $Y$, Omotara B A, Iganus R B, Manzi F. Why not bathe the baby today?: A qualitative study of thermal care beliefs and practices in four African sites. BMC Pediatr. $2015 ; 15: 156$.

13. Mariko D. Connaissances relatives aux soins essentiels du nouveau-né du personnel du centre de santé de référence de la commune $\mathrm{VI}$ du district de Bamako. Thèse méd, Université de Bamako-Mali, FMOS, 2018, 74-76 p.

14. Njom Nlend A E, Beyeme M. Audit des pratiques d'accueil et des soins essentiels du nouveau-né dans les centres de santé de premier niveau à Yaoundé. Journal de Pédiatrie et Puériculture, 2015 ; 4:190-194.

15. Dicko Traore F, Gire C, Brevaut Alaty V, Busutill M, Monnier A S, Brunel V. Evaluation des connaissances et pratiques d'hygiène des mains au niveau de l'unité de néonatologie et soins intensifs du service de médecine infantile et néonatologie de l'hôpital Nord Marseille. Journal de Pédiatrie et Puériculture, 2011 ; 2: 172-177. 
16. Jyoti S, Jeeva S, Geetanjli Poonam S. Practices of Auxiliary Nurse Midwives Regarding Care of Baby at Birth. Nursing and Midwifery Research Journal, $2011 ; 7,110-119$.

17. Mariko O. Etude sur les connaissances, attitudes et pratiques des femmes sur l'allaitement maternel exclusif dans le quartier de Sogoniko en commune 6 du District de Bamako. Thèse Méd, Université de Bamako-Mali, FMPOS, N 10M380, 2010, 97-98 p.

18. Gamgne B K. Etude des connaissances et pratiques des mères sur l'allaitement maternel à l'hôpital provincial et à la PMI de Bafoussam. Thèse méd. Bafoussam-Cameroun : institut supérieur des sciences et de la santé Bangangté, 2009.

19. Monebenimp F, Tenefopa M, Mve Kok V, Kago I. Competence of health care providers on care of newborns at birth in a level-1 health facility in Yaoundé, Cameroun. Pan African Medical Journal, 2012 ; $11: 45$.

Liste des tableaux :

Tableau I : Répartition des enquêtées selon la formation reçue en SENN

\begin{tabular}{lcc}
\hline Formation reçue sur les SENN & Première évaluation & Deuxième évaluation \\
\cline { 2 - 3 } & Effectif & Effectif \\
\hline Oui & 6 & 8 \\
Non & 2 & 0 \\
Total & 8 & 8 \\
\hline
\end{tabular}

Tableau II : Répartition des enquêtées selon le nombre d'années d'exercice

\begin{tabular}{lc}
\hline Nombre d'années d'exercice & Effectif \\
\hline Moins 1 an & 0 \\
1 à 5 ans & 3 \\
6 à 10 ans & 1 \\
11 à 15 ans & 1 \\
16 à 20 ans & 1 \\
Plus de 20 ans & 2 \\
Total & 8 \\
\hline
\end{tabular}

Tableau III : Pratiques du personnel relatives aux étapes de soins immédiats

\begin{tabular}{lcc} 
Pratiques du personnel relatives aux étapes & Première évaluation & Deuxième évaluation \\
\cline { 2 - 3 } de soins immédiats & Effectif & Effectif \\
\hline Etape 1 & 8 & 8 \\
Etape 2 & 8 & 8 \\
Etape 3 & 8 & 8 \\
Etape 4 & 8 & 8 \\
Etape 5 & 8 & 8 \\
Etape 6 & 2 & 8 \\
Etape 7 & 8 & 8 \\
Etape 8 & 8 & 8 \\
Etape 9 & 2 & 8 \\
Etape 10 & 8 & 8 \\
Etape 11 & 4 & $\mathbf{8}$ \\
Total & $\mathbf{8}$ & 8 \\
\hline
\end{tabular}


Tableau IV : Pratiques du personnel relatives aux soins aux nouveau-nés au cours des six premières heures après la naissance

\begin{tabular}{lcc}
\hline Pratiques relatives aux soins aux nouveau-nés au cours & Première évaluation & \multicolumn{2}{c}{ Deuxième évaluation } \\
\cline { 2 - 3 } des six 1iers heures après la naissance & Effectif & Effectif \\
\hline Respiration & 0 & 8 \\
Température & 0 & 8 \\
Saignement Ombilical & 0 & 8 \\
Coloration & 0 & 8 \\
Bonne pratique d'allaitement maternel vérifiée & 0 & 8 \\
Examen Complet avant la sortie & 2 & 8 \\
\hline
\end{tabular}

Tableau V : Répartition des mères selon la tranche d'âge

\begin{tabular}{lcc}
\hline Tranche d'âge & Première évaluation & Deuxième évaluation \\
\cline { 2 - 3 } & Effectif & Effectif \\
\hline $15-19$ ans & 0 & 2 \\
$20-24$ ans & 10 & 10 \\
$25-49$ ans & 6 & 4 \\
Total & 16 & 16 \\
\hline
\end{tabular}

Tableau VI : Pratiques du personnel relatives aux consignes à dicter à la mère à la sortie (Signes de danger)

\begin{tabular}{lcc} 
Signes de danger & Première évaluation & Deuxième évaluation \\
\cline { 2 - 3 } & Effectif & Effectif \\
\hline Oui & 0 & 16 \\
Non & 16 & 0 \\
Total & 16 & 16 \\
\hline
\end{tabular}

